\title{
Fundamental frequency contour modeling using HMM and categorical multiple regression technique
}

\author{
Toshiaki Fukada, ${ }^{\dagger}$ Yasuhiro Komori, Takashi Aso, \\ and Yasunori Ohora \\ Media Technology Laboratory, Canon Inc., \\ 890-12, Kashimada, Saiwai-ku, Kawasaki, 211 Japan
}

(Received 30 November 1994)

\begin{abstract}
This paper proposes a novel fundamental frequency $\left(F_{0}\right)$ contour modeling based on statistics aiming at text-to-speech. In the proposed modeling, the $F_{0}$ contour of a sentence is constructed by statistical minor phrase models. These models consist of a sophisticated integration of local models of normalized pitch patterns and global models of maxima and dynamic ranges. Hidden Markov Model (HMM) is introduced to determine the normalized pitch patterns (pitch-HMM). To determine the maximum and the dynamic range, categorical multiple regression technique (CMRT) is introduced. HMM is a good statistical model which directly represents the $F_{0}$ contours by several reliable states. Moreover, it is easy to take relative changes of the $F_{0}\left(\Delta F_{0}\right)$ and phonetic environments into account. CMRT is a good statistical modeling technique which is able to deal with syntactic structures and acoustic events in a sentence simultaneously. Evaluation on the pitch-HMMs shows accent type identification rate of $91 \%$ and RMS error of $9.2 \mathrm{~Hz}$. Evaluation on the maximum and the dynamic range models gives 0.901 and 0.835 for the multiple correlation coefficients, respectively. Finally, the result of the subjective evaluation indicates that the proposed modeling is superior to the conventional modeling.
\end{abstract}

Keywords: Fundamental frequency, HMM, Categorical multiple regression technique, Minor phrase, Speech synthesis

PACS number: 43. 72. Ja

\section{INTRODUCTION}

A good generation model of fundamental frequency $\left(F_{0}\right)$ contours is essential for speech synthesis. Recently, several methods of $F_{0}$ contour modeling based on statistical analysis have been proposed. ${ }^{1-4)}$

One approach, $F_{0}$ contour modeling based on two-stage strategy, ${ }^{2,3)}$ consists of a global model and a local model. These models are directly generated from $F_{0}$ contours without separating the $F_{0}$ into accentual components and phrase components. The global model characterizes global positions of $F_{0}$

\footnotetext{
$\uparrow$ Present address: ATR Interpreting Telecommunications Research Laboratories, 2-2, Hikaridai, Seikacho, Soraku-gun, Kyoto, 619-02 Japan
}

shape of minor phrases such as the maximum $F_{0}$ values, and the local model characterizes local $F_{0}$ movements inside each minor phrase. Both studies apply the categorical multiple regression technique (henceforth CMRT) to generate the global model, but their methods of local modeling differ. Abe proposed a local model generated from the average $F_{0}$ and difference $F_{0}$ values of vowel parts in each syllable using CMRT. ${ }^{2}$ However, this model is insufficient for capturing the local $F_{0}$ movements in a syllable (such as rise-fall or fall-rise) because only a single point pitch for each syllable is given to represent $F_{0}$ contour. Iwata used a local model ("accentual phrase pitch pattern models" defined by Iwata) generated from three fixed points of $F_{0}$ 
in each vowel part.5) The idea to represent the local movements of $F_{0}$ contour by several point pitches had an advantage compared to Abe's approach. However, there should be more reliable modeling, which suits the local movements of $F_{0}$ contour, than the fixed point modeling.

Hidden Markov Model (HMM) is well-known as a powerful method which deals with such a requirement. HMM is a good statistic model which is able to directly represent the $F_{0}$ contours by several reliable states. Moreover, it is able to use relative changes of $F_{0}\left(\Delta F_{0}\right)$ and is able to consider the phonetic environments. However, the $F_{0}$ contour modeling based on HMM has been proposed on the study of speech recognition for word accent identification ${ }^{6)}$ and phrase boundary detection, ${ }^{7)}$ while there are only a few studies of speech synthesis. Ljolje tried to generate the $F_{0}$ contours using a prosodic HMM. ${ }^{8}$ However his method cannot be applied directly to a text-to-speech system, because his approach was tested only on monosyllabic words.

Based on these researches, we propose here a novel statistical $F_{0}$ contour modeling aiming at textto-speech. In the proposed modeling, the $F_{0}$ contour of a sentence is constructed by statistic minor phrase models. These models consist of a sophisticated integration of local models of normalized pitch patterns and global models of a maximum and a dynamic range. HMM is introduced to determine the normalized pitch patterns (pitch-HMM). To determine the maximum and the dynamic range, CMRT is introduced, which is a good statistic method to deal with syntactic structures and acoustic events in a sentence simultaneously.

In the following sections, we first describe the proposed statistical $F_{0}$ contour modeling. These models are evaluated in section 3 and 4 . Section 5 shows the result of listening tests. Section 6 discusses some important issues concerning the pitchHMM.

\section{2. $\boldsymbol{F}_{0}$ CONTOUR MODELING}

$2.1 F_{0}$ Contour Modeling using Pitch-HMMs, Maximum and Dynamic Range Models

The $F_{0}$ contour of a minor phrase, $f(t)$, which constitutes a sentence is represented by the following equation:

$$
f(t)=d \cdot p(t)+m-d,
$$

where $p(t)$ is the normalized pitch pattern generated from normalized point pitch models; $m$ is the maximum of $f(t)$ and $d$ is the dynamic range, i.e. the difference between the maximum and the minimum of $f(t)$, calculated for each minor phrase. We generate $f(t)$ through the normalized point pitch models using HMM technique (pitch-HMMs) and the maximum and the dynamic range models using CMRT.

\subsection{Pitch-HMMs}

Pitch-HMMs are obtained from a large scale database consisting of prosodic features, segmented phoneme boundaries, and lexical information (i.e. mora count and accent type). $F_{0}$ and differenced fundamental frequency (henceforth $\Delta F_{0}$ ) are used as prosodic features. Here, since raw $F_{0}$ values $f(t)$ vary widely among minor phrases, $F_{0}$ is normalized as follows:

$$
p(t)=(f(t)-m+d) / d,
$$

where $m$ and $d$ are the maximum and the dynamic range of $f(t) . \Delta F_{0}$ is computed from $p(t)$ by linear regression.

The pitch-HMMs are trained by considering phonetic environments (e.g. accent type, mora count, mora position, phonemic category, etc.). Therefore, the pitch-HMMs can express the difference of the $F_{0}$ contours related to phonemes. ${ }^{9)}$ This is meaningful because it is reported that expressing the microscopic movements in a phoneme improves the intelligibility of a synthesized speech. ${ }^{10}$ ) These movements in the $F_{0}$ contours can be captured by the states in the pitch-HMM.

The $F_{0}$ contour of each minor phrase $f(t)$ is generated by the following steps (See Fig. 1; the example is "/azi/(taste)".).

1. Select the pitch-HMMs according to the phonetic environments (Fig. 1(a)).

2. Align the pitch-HMMs according to the segmental durations (Fig. 1(b)).

3. Determine the target point pitches and generate the normalized pitch pattern by interpolating these points (Fig. 1(c)).

4. Multiply with the dynamic range and add the bias (Fig. 1(d)):

$$
f(t)=d \cdot p(t)+m-d .
$$

Details for the pitch-HMM alignment and the normalized pitch pattern generation are described in section 3.2.2. Furthermore, advanced techniques 


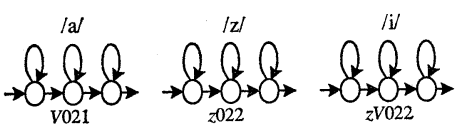

(a) Desirable pitch-HMM selection. The V021 means that phonemic category, accent type, mora count and current mora position are vowel, 0,2 and 1 , respectively.

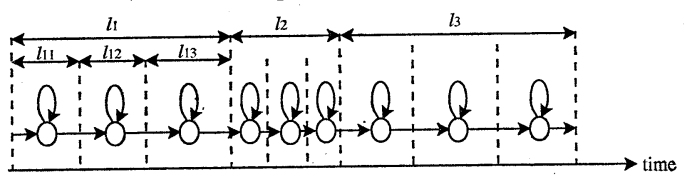

(b) Alignment of the pitch-HMMs.

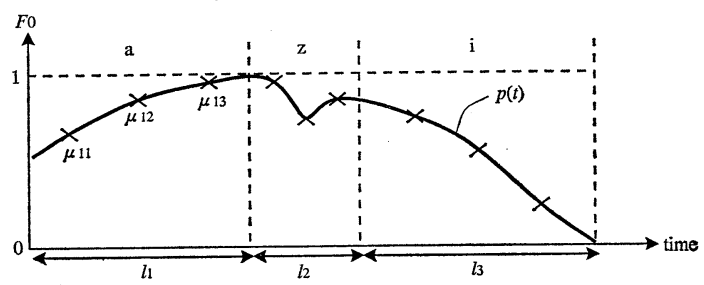

(c) Normalized pitch pattern $p(t)$.

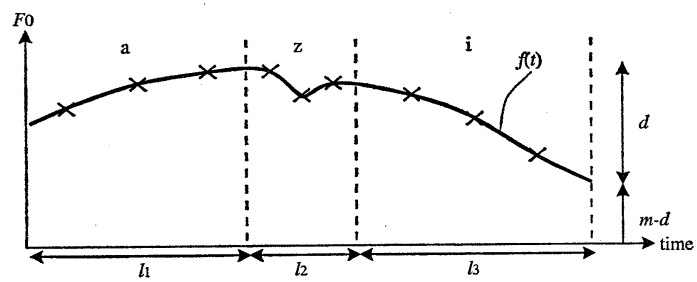

(d) $F_{0}$ contour $f(t)$.

Fig. 1 Conceptual figure of $F_{0}$ contour generation.

for the alignment and the generation are discussed in section 6 .

\subsection{Maximum and Dynamic Range Models}

We apply $\mathrm{CMRT}^{11)}$ for the maximum and the dynamic range modeling. The maximum $m$ of a minor phrase is predicted by the following equation:

$$
P_{i}=\sum_{j=1}^{R} \sum_{k=1}^{C_{j}} \alpha_{j k} \delta_{i}(j k), \quad(i=1, \cdots, N)
$$

where $P_{i}$ is the predicted value of the $i$-th sample, $R$ is the number of factors and $C_{j}$ is the number of categories of a factor $j . \quad \delta_{i}(j k)$ is the characteristics function defined as

$$
\delta_{i}(j k)= \begin{cases}1, & \begin{array}{l}
\text { if the } i \text {-th sample falls into } \\
\text { the category } k \text { of factor } j,
\end{array} \\
0, & \text { otherwise }\end{cases}
$$

$a_{j k}$ is obtained by minimizing the summation of the prediction error $E$ :

$$
E=\sum_{i=1}^{n}\left(p_{i}-P_{i}\right)^{2},
$$

where $p_{i}$ is the maximum of the $i$-th sample.

The dynamic range is predicted in an equivalent way.

\subsection{Advantages}

The proposed modeling has the following advantages. As for HMM:

- Thanks to the ability of time-warping and training with the maximum likelihood criterion, reliable point pitches are automatically determined.

- Multiple prosodic parameters $\left(e . g . F_{0}\right.$ and $\left.\Delta F_{0}\right)$ can be used, directly and simultaneously, which makes the model more stable.

- By taking account of phonetic environments (e.g. accent type, mora count, mora position, phonemic category, etc.), precise models can be obtained.

- Detailed movements in the $F_{0}$ contours can be captured by using larger number of HMM states.

To demonstrate the advantage of HMM based modeling, consider the three simple contours $\left(F_{1}(t), F_{2}(t), F_{3}(t)\right)$ shown in Fig. 2. We compare the following three modeling approaches to calculate representative point pitches $\left(f_{1}, f_{2}, f_{3}\right)$ :

1. mean values sampled at the positions of $t=$

$$
\begin{aligned}
& 1 / 6, t=3 / 6 \text { and } t=5 / 6 \\
& \text { (e.g. } \left.f_{1}=(1 / 3) \sum_{m=1}^{3} F_{m}(1 / 6)\right),
\end{aligned}
$$

2. mean values averaged between $t=0$ and $t=$ $1 / 3$, between $t=1 / 3$ and $t=2 / 3$, and between $t=2 / 3$ and $t=1$ (e.g. $\left.f_{1}=(1 / 3) \sum_{m=1}^{3} \int_{0}^{1 / 3} F_{m}(t) d t\right)$,

3. pitch-HMMs consisting of three states with a single Gaussian density function.

Three point pitches are respectively determined:

1. $f_{1}=0.36, f_{2}=0.83, f_{3}=0.36(d y n=0.71)$,

2. $f_{1}=0.36, f_{2}=0.78, f_{3}=0.36(d y n=0.63)$,

3. $f_{1}=0.39, f_{2}=0.89, f_{3}=0.39(d y n=0.75)$,

where dyn indicates the dynamic range value, obtained by interpolation of these point pitches using a straight line between $t=0$ and $t=1$. It is seen from these results that dyn of HMM base modeling is the nearest value to the original dynamic range $(d y n=1.0)$.

CMRT is able to deal with syntactic structure (e.g. parts of speech and dependency structure) and 


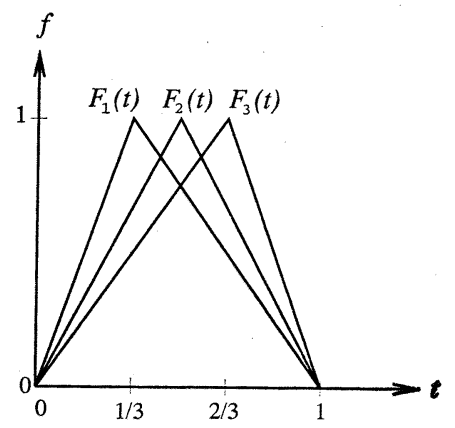

Fig. 2 Examples of contours.

acoustic events (e.g. accent type and mora count) in a sentence simultaneously. It facilitates to control the dynamics of the pitch-HMMs.

\section{EVALUATION OF PITCH-HMMS}

In this section, we evaluate the pitch-HMMs through two experiments. One is an accent identification experiment for investigating how appropriate the pitch-HMMs express the accent types. The other is an average of RMS error measurement for comparing the difference between the original $F_{0}$ and the one generated from the pitch-HMMs.

\subsection{Conditions}

For training and evaluation of the pitch-HMMs, we used the ATR 5,240 word database sampled at $12 \mathrm{kHz}^{12)}$ uttered by one male speaker. The database contained phoneme boundary information. The lists of accent types and mora counts for these words were also prepared.

The $F_{0}$ contours were estimated by FFT cepstral analysis, with an $F_{0}$ value for every $2.5 \mathrm{~ms}$. The $F_{0}$ was warped on a logarithmic scale, and then median smoothed. Additionally, half-pitch and doublepitch errors were corrected automatically. Hand correction of extraction errors was not performed, but the parameters for the smoothing algorithms were tuned to the database. The first regression coefficients of the $F_{0}\left(\Delta F_{0}\right)$ were estimated with a regression window of $77.5 \mathrm{~ms}$.

Accent type, mora count, mora position and phonemic category were considered as phonetic environments. For $i$ morae and $j$ classes of phonemic category, the number of pitch-HMMs $N=i^{2} j$. Pitch-HMMs, consisting of three states with a single Gaussian density function, were trained by
Table 1 Types of phonemic category.

\begin{tabular}{ll}
\hline Model & \multicolumn{1}{c}{ Class of pitch-HMMs } \\
\hline $\begin{array}{l}\text { Rough } \\
\text { (2 classes) }\end{array}$ & $\begin{array}{l}\text { vowels: *V } \\
\text { voiced consonants: * }\end{array}$ \\
\hline Detailed & vowels: V (no consonant), \{ptk\}V, \\
(15 classes) & $\{$ fsh $\} \mathrm{V},\{$ bdg $\} \mathrm{V},\{\mathrm{mn}\} \mathrm{V}, \mathrm{rV}, \mathrm{wV}, \mathrm{yV}$, \\
& $\mathrm{zV}$ \\
& voiced consonants: $\{$ bdg $\},\{\mathrm{mn}\}, \mathrm{r}$, \\
& w, y, z \\
\hline " $\{$ ptk $\}$ V" indicates any vowel preceded by any of the
\end{tabular}
consonants "p," "t," or "k."

the Forward-Backward algorithm. ${ }^{13)}$

As for the pitch-HMMs, we selected two HMM sets: one consisting of two classes of phonemic category (rough models) and the other of 15 classes of phonemic category (detailed models), as shown in Table 1. We expect that the precision of $F_{0}$ countour estimation improves by increasing the number of the pitch-HMMs according to the phonemic environments, as it has been reported that $F_{0}$ contours differ according to the phonemic environments, even if the words have same accent type and same mora count. ${ }^{9)}$

\subsection{Results}

3.2.1 Accent identification experiment

The appropriateness of the pitch-HMMs was evaluated by an accent identification experiment using four mora words (1,565 words) in the 5,240 word database. In this experiment, the rough pitch-HMM set was adopted and speech data with devocalized vowel and with concatenated phoneme labels were not considered.

This experiment was simply performed using the vowel parts. The accent pattern models were created by concatenating the null code model, which forces mora alignment, in between the rough pitchHMMs (see Fig. 3). As for test data, $F_{0}$ and $\Delta F_{0}$ sequences of vowel parts were extracted by using the label information, and null data was also inserted

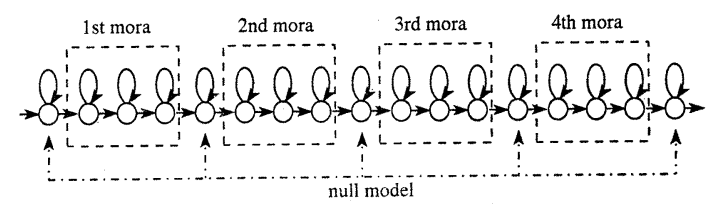

Fig. 3 Pitch-HMM concatenation. 


\section{T. FUKADA et al.: STATISTICAL $F_{0}$ CONTOUR MODELING}

Table 2 Confusion matrix of accent identification of 4 mora words. The pitchHMMs were trained by using two features, $F_{0}$ and $\Delta F_{0}$.

\begin{tabular}{crrrrr}
\hline $\begin{array}{c}\text { Accent } \\
\text { type }\end{array}$ & 0 & 1 & 2 & 3 & ID rate \\
\hline 0 & 921 & 4 & 50 & 76 & $87.6 \%$ \\
1 & 0 & 143 & 4 & 0 & $97.3 \%$ \\
2 & 3 & 0 & 78 & 6 & $89.7 \%$ \\
3 & 4 & 0 & 20 & 256 & $91.4 \%$ \\
\hline
\end{tabular}

Total ID rate: $91.5 \%$.

Table 3. Confusion matrix of accent identification of 4 mora words. The pitchHMMs were trained by using one features, $F_{0}$.

\begin{tabular}{crrrrr}
\hline $\begin{array}{c}\text { Accent } \\
\text { type }\end{array}$ & 0 & 1 & 2 & 3 & ID rate \\
\hline 0 & 862 & 6 & 44 & 139 & $82.0 \%$ \\
1 & 2 & 144 & 1 & 0 & $98.0 \%$ \\
2 & 11 & 0 & 65 & 11 & $74.7 \%$ \\
3 & 48 & 0 & 22 & 210 & $75.0 \%$ \\
\hline
\end{tabular}

Total ID rate: $82.4 \%$.

between each vowel part. This forced alignment allows explicit usage of the duration information in the database. The Viterbi beam search algorithm ${ }^{13)}$ was adopted for the evaluation.

Table 2 shows the experimental result using $F_{0}$ and $\Delta F_{0}$ as prosodic parameters. For comparison, the result using only $F_{0}$ is shown in Table 3. The drastic improvement from $82.4 \%$ to $91.5 \%$ accuracy indicates the effectiveness of the additional $\Delta F_{0}$ for the pitch-HMMs. This result shows that the pitch-HMMs trained using $F_{0}$ and $\Delta F_{0}$ express accent patterns more appropriately.

\subsubsection{RMS error measurement}

We measured an average of RMS error between the original $F_{0}$ contours and the one generated from the pitch-HMMs using the 5,240 words. The original values of the phoneme durations $\left(l_{1}, l_{2}\right.$ and $l_{3}$ in Fig. 1(b)), the maxima and the dynamic ranges ( $m$ and $d$ in Fig. 1(d)) were given to the pitchHMMs.

In this experiment, the pitch-HMMs were simply aligned at the positions of $1 / 6,3 / 6$ and $5 / 6$ of each phoneme duration (i.e. $l_{11}=l_{12}=l_{13}=l_{1} / 3$ in Fig. 1(b)). The $F_{0}$ mean values of pitch-HMMs were used for the target point pitches $\left(\mu_{11}, \mu_{12}, \mu_{13}\right.$, etc. in
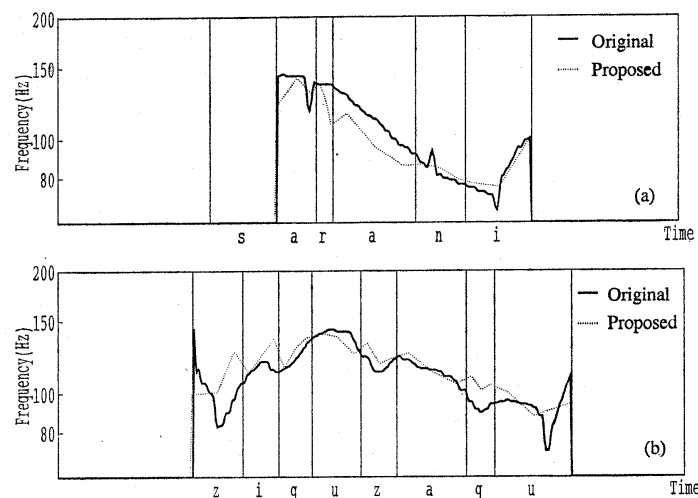

Fig. 4 Examples of the $F_{0}$ contour generation using the rough models. (a) "/sarani/ (moreover)," (b) "/ziguzagu/ (zigzag)."
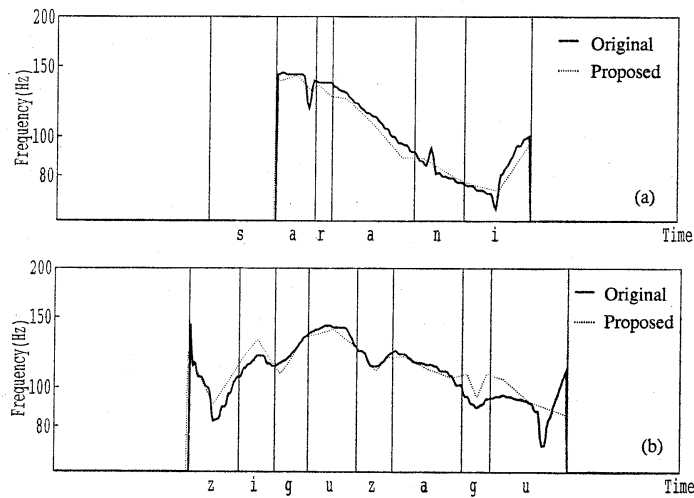

Fig. 5 Examples of the $F_{0}$ contour generation using the detailed models.

Fig. 1(c)) and a simple straight line interpolation was adopted. We discuss a more reliable state alignment and a generation of the normalized pitch pattern in section 6 .

As a result, the RMS error using the rough models was 1.46 semitone (equivalent to $10.2 \mathrm{~Hz}$ at $120 \mathrm{~Hz}$ ). Figure 4 shows the examples using the rough models. The RMS error using the detailed models improved to 1.32 semitone (equivalent to $9.2 \mathrm{~Hz}$ at $120 \mathrm{~Hz}$ ). This is about $10 \%$ improvement from the rough models. Figure 5 shows the examples using the detailed models. As shown in Fig. 5 , the $F_{0}$ contours were well estimated in comparison with Fig. 4. It is also seen that the microscopic $F_{0}$ movement corresponding to a phoneme is well captured (see both /z/'s in Fig. 5(b)). 


\section{EVALUATION OF MAXIMUM AND DYNAMIC RANGE MODELS}

In this section, we evaluate the maximum and the dynamic range models generated using CMRT.

\subsection{Conditions}

The maximum and the dynamic range were computed from the minor phrases in the ATR 503 sentence database. ${ }^{12)}$ As for test data, 43 sentences in another ATR database were used. These databases were uttered by the same speaker.

The $F_{0}$ contours were estimated in the same manner as described in section 3.1. The maximum and the dynamic range of each minor phrase were calculated from the $F_{0}$ contours on a logarithmic scale.

The factors and the numbers of categories for the maximum (Max) and the dynamic range (Dyn) models are shown in Table 4. Difference between mora count and accent type (i.e. mora count after the accentual mora) was selected because we have found from a preliminary experiment that the variance of $F_{0}$ dynamic range categorized by this factor was smaller than that categorized by accent type. The boundary environment means the combination of the preceding type (beginning of sentence, pause, continuation) and the succeeding type (end of sentence, pause, continuation). In order to smooth a potential gap between minor phrases, the preceding maximum and minimum values were utilized for the maximum model and the current maximum value for the dynamic range model.

\subsection{Results}

The partial correlation coefficients of both models are shown in Table 4. As shown in this table, the boundary environment and the mora count played important roles in predicting the maximum. The maximum and the minimum of the preceding minor phrase for the maximum model were also effective. For the dynamic range model, the maximum of the current minor phrase was the second most significant factor.

Table 5 shows the multiple correlation coefficients of both models. It is seen from this table that high multiple correlation coefficients for both models are obtained, i.e. both models are good prediction models. The averages of the RMS errors for close and open data are also shown in this table. The
Table 4 Factors, number of categories and partial correlation coefficients.

\begin{tabular}{|c|c|c|c|c|}
\hline \multirow[t]{2}{*}{ Factor } & \multicolumn{2}{|c|}{$\begin{array}{l}\text { Number of } \\
\text { categories }\end{array}$} & \multicolumn{2}{|c|}{$\begin{array}{l}\text { Partial } \\
\text { correlation } \\
\text { coefficients }\end{array}$} \\
\hline & Max & Dyn & $\operatorname{Max}$ & Dyn \\
\hline $\begin{array}{l}\text { Position of first mora of } \\
\text { minor phrase within a } \\
\text { major phrase }\end{array}$ & 8 & 5 & 0.173 & 0.090 \\
\hline Mora count & 6 & 9 & 0.377 & 0.212 \\
\hline Parts of speech & 10 & 10 & 0.240 & 0.131 \\
\hline $\begin{array}{l}\text { Dependency structure } \\
\text { (from preceding) }\end{array}$ & 2 & 2 & 0.246 & 0.029 \\
\hline $\begin{array}{l}\text { Dependency structure } \\
\text { (to succeeding) }\end{array}$ & 2 & 2 & 0.112 & 0.005 \\
\hline $\begin{array}{l}\text { Difference between mora } \\
\text { count and accent type } \\
\text { (preceding) }\end{array}$ & 7 & 7 & 0.352 & 0.225 \\
\hline $\begin{array}{l}\text { Difference between mora } \\
\text { count and accent type } \\
\text { (current) }\end{array}$ & 8 & 8 & 0.271 & 0.648 \\
\hline Accent type (succeeding) & 3 & 3 & 0.047 & 0.065 \\
\hline Boundary environment & 8 & 8 & 0.439 & 0.281 \\
\hline Maximum (preceding) & 10 & - & 0.209 & - \\
\hline Minimum (preceding) & 10 & - & 0.140 & - \\
\hline Maximum (current) & - & 9 & - & 0.496 \\
\hline
\end{tabular}

Table 5 Multiple correlation coefficients and RMS error.

\begin{tabular}{cccc}
\hline \multirow{2}{*}{ Model } & \multirow{2}{*}{$\begin{array}{c}\text { Multiple } \\
\text { correlation } \\
\text { coefficients }\end{array}$} & \multicolumn{2}{c}{ RMS error semitone $(\mathrm{Hz})$} \\
\cline { 3 - 4 } & 0.901 & $1.77(12.22)$ & $2.44(16.89)$ \\
Max & 0.835 & $2.13(14.76)$ & $2.38(16.50)$ \\
\hline
\end{tabular}

RMS errors of the maximum and the dynamic range models for close data came up to 1.77 semitone (equivalent to $12.22 \mathrm{~Hz}$ at $120 \mathrm{~Hz}$ ) and 2.13 semitone $(14.76 \mathrm{~Hz})$, respectively. As for the open data, the RMS errors of both models came up to 2.44 semitone $(16.89 \mathrm{~Hz})$ and 2.38 semitone $(16.50 \mathrm{~Hz})$. The small difference between the results of the close and the open indicates that robust models are obtained.

\section{SUBJECTIVE EXPERIMENTS}

To evaluate the generated $F_{0}$ contours, two kinds of subjective evaluation were carried out. The first experiment (Exp. 1) was designed to evaluate the performance of the pitch-HMMs, and the second (Exp. 2) was to evaluate the total performance of 


\section{T. FUKADA et al: : STATISTICAL $F_{0}$ CONTOUR MODELING}

the proposed modeling.

\subsection{Conditions}

In both experiments, the stimuli were generated by the cepstral synthesis-analysis method. ${ }^{14)}$ The following three kinds of $F_{0}$ contours were prepared for comparison:

- extracted from natural speech (Original)

- generated from the proposed model (Proposed)

- generated from a conventional model ${ }^{15}$ (Conventional).

In the conventional model, stress level of accent component was set to 4.0 semitone and phrase component was set to 0.4 semitone/mora. Note that a single stress level was used in this experiment, whereas Hakoda adopted five stress levels ${ }^{15}$ ) The same durations and cepstral coefficients were given to the three stimuli.

In Exp. 1, 24 minor phrases were used as stimuli. In order to compare the pitch-HMMs with the conventional point pitches, the original maximum and the original dynamic range were given to the pitchHMMs and to the conventional model. In Exp. 2, 20 sentences were used as stimuli. These stimuli were evaluated by a subjective opinion test of five grades with 14 listeners. The grades were "very good," "good," "fair," "bad," and "very bad," and the score points were 5, 4, 3, 2 and 1 , respectively.

The pitch-HMMs and the maximum and the dynamic range models were generated using the ATR 503 sentence database. The number of the phonemic categories was set to two (rough models) allowing for the trainability of the pitch-HMMs. Most of the conditions were the same as described in section 3.1 except prosodic parameters: a relative position in a phoneme was additionally used to $F_{0}$ and $\Delta F_{0}$. This parameter was introduced considering the following:

- Variances of $F_{0}$ contours of sentence utterances are larger than those of word utterances owing to the boundary environment, the syntactic dependency structure, etc.

- The pitch-HMMs are aligned at the fixed positions in our current version.

From a preliminary experiment, we confirmed that the pitch-HMMs trained using $F_{0}, \Delta F_{0}$ and relative positions had perceptually better quality than those trained using only $F_{0}$ and $\Delta F_{0}$.

\subsection{Results}

The result of Exp. 1 is shown in Fig. 6(a). The Mean Opinion Score (MOS) of the pitch-HMMs was 3.5 , while the score of the conventional point pitch model was 3.2. Moreover, no listener had a higher MOS for the conventional model than for the pitch-HMM. These results demonstrate the effectiveness of the pitch-HMMs.

Figure 6(b) shows the result of Exp. 2. The scores of the proposed and the conventional models were 3.8 (close to "good") and 2.2 (close to "bad") respectively. The proposed modeling is significantly superior to the conventional modeling and the proposed models can generate high quality $F_{0}$ contours. As for this improvement, we believe that the global $F_{0}$ shape is playing a major role in the performance of the proposed modeling.

One word of caution: in this experiment, the proposed model was compared to the simplest conventional model (i.e. single stress level was used). To prove the superiority of the proposed method against the conventional methods, more sophisticated stimuli should be used.

Figure 7 shows an example of $F_{0}$ contour of open data; (a) waveform, (b) the original $F_{0}$ contour, (c) the generated $F_{0}$ contour by the proposed model. It is seen from this figure that the global $F_{0}$ features of each minor phrase (i.e. the maximum and the dynamic range) are well estimated. The detail movements of the $F_{0}$ contour are also well captured (e.g. ihe $F_{0}$ shapes (rise-fall) at the 1 st and the 4th minor phrases are captured appropriately in spite of their accent type being type 1 (fall)).

Pitch-HMMs for up to 8 morae can be trained from the 503 sentence database. Considering application of the pitch-HMMs to a text-to-speech system, it is required to cover the actual minor phrase patterns in the real world. We investigated

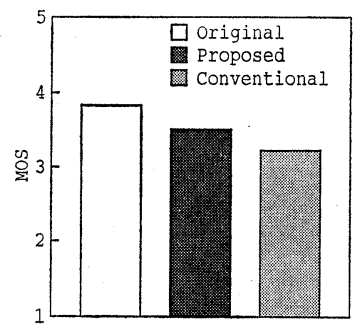

(a) minor phrase

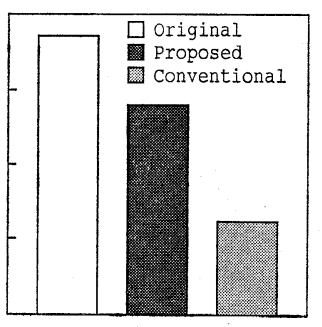

(b) sentence
Fig. 6 Subjective evaluation results. 


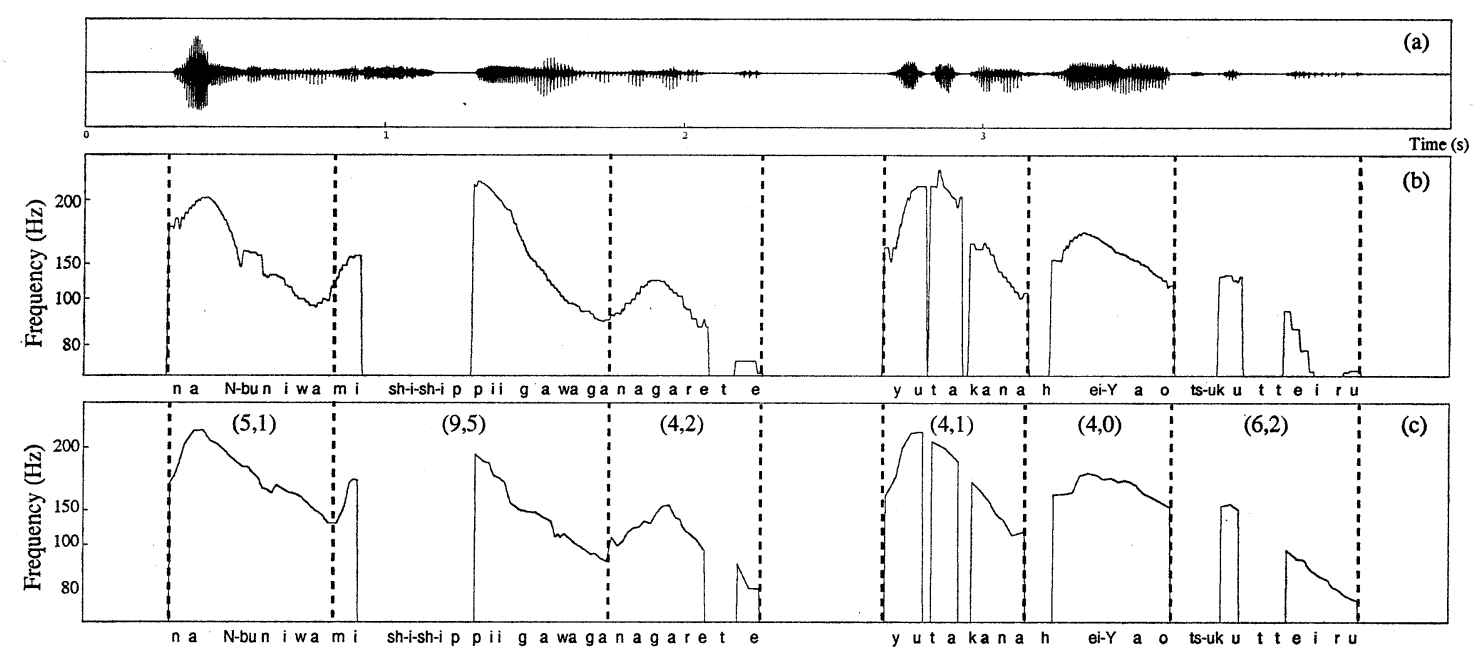

Fig. $7 F_{0}$ sentence contours using HMM and CMRT: (a) waveform, (b) original $F_{0}$ contour, (c) estimated $F_{0}$ contour generated by the proposed model. This sentence consists of 6 minor phrases. $(5,1)$ indicates the mora count and the accent type.

the coverage using 2.3 Megabyte of text corpora including many areas. As a result, the minor phrases for 8 morae or less accounted for $94.1 \%$ coverage in the corpora. The result indicates that the trained pitch-HMMs cover the real world adequately. As for the rest of $5.9 \%$, the conventional point pitch model or integration of the pitch-HMMs and the conventional model could be utilized.

\section{DISCUSSION}

In this section, we discuss some important issues on the pitch-HMM, particularly the state alignment and the way to generate the normalized pitch pattern.

\subsection{Alignment}

In the previous experiments, the pitch-HMMs were simply aligned at the positions of $1 / 6,3 / 6$ and $5 / 6$ in each phoneme. However, it should be more desirable to align the three states of the pitch-HMM appropriately by taking account of their own statistical information (i.e. mean, variance, output probabilities and transition probabilities). The statistical durations of the HMM states can be obtained from the Viterbi decoding algorithm. ${ }^{13)}$ When a phoneme duration is given, the duration of each of the three states (i.e. $l_{11}, l_{12}$ and $l_{13}$ in Fig. 1(b)) can be determined according to the ratio of the obtained statistical durations.

\subsection{Interpolation}

As for the interpolation, although the simple straight line interpolation was adopted in the previous experiments, various interpolating techniques (e.g. spline interpolation or Bézier curve) can be applied for making the $F_{0}$ contours smoother.

\subsection{Pitch Pattern Generation}

As for the normalized pitch pattern generation, the time series of $F_{0}$ can be computed by using the statistical information of the pitch-HMMs, in terms of maximizing the log-likelihood. For simple explanation, let us consider generating $F_{0}$ contour from a single state pitch-HMM (e.g. state $s(s=1,2$, $3)$ ). If the duration of the pitch-HMM, $l$, is given, a time series of normalized pitches, $\boldsymbol{O}_{1}=\left[O_{1}(1)\right.$, $\left.O_{1}(2), \ldots, O_{1}(l)\right]$, can be determined as follows.

The likelihood of $F_{0}, f\left(\boldsymbol{O}_{1}\right)$, with respect to $\boldsymbol{O}_{1}$ is:

$$
f\left(\boldsymbol{O}_{1}\right)=\prod_{t=1}^{l}\left(a_{1}\right)^{l-1} b_{1}\left(O_{1}(t)\right) \cdot\left(1-a_{1}\right),
$$

where $a_{1}$ is a self-loop transition probability in the state $s$ and $b_{1}\left(O_{1}(t)\right)$ is an output probability. The output probability of a Gaussian probability density function is:

$$
b_{1}\left(O_{1}(t)\right)=\frac{1}{\sqrt{2 \pi \sigma_{1}^{2}}} \exp \left\{-\frac{\left(O_{1}(t)-\mu_{1}\right)^{2}}{2 \sigma_{1}^{2}}\right\},
$$

where $\mu_{1}$ and $\sigma_{1}^{2}$ are the mean and the variance of $F_{0}$ of the pitch-HMM. Similarly, the likelihood of 
the $\Delta F_{0}, g\left(\boldsymbol{O}_{2}\right)$ is represented by a set of $\Delta F_{0}\left(\boldsymbol{O}_{2}=\right.$ $\left.\left[O_{2}(1), O_{2}(2), \ldots, O_{2}(l)\right]\right)$, the $\Delta F_{0}$ mean $\left(\mu_{2}\right)$ and the $\Delta F_{0}$ variance $\left(\sigma_{2}^{2}\right)$.

Therefore, the $\log$-likelihood $L$ is:

$$
\begin{aligned}
L= & \log f\left(\boldsymbol{O}_{1}\right)+\log g\left(\boldsymbol{O}_{2}\right) \\
= & \sum_{t=1}^{l}\left\{-\frac{\left(O_{1}(t)-\mu_{1}\right)^{2}}{2 \sigma_{1}{ }^{2}}\right\} \\
& +\sum_{t=1}^{l}\left\{-\frac{\left(O_{2}(t)-\mu_{2}\right)^{2}}{2 \sigma_{2}{ }^{2}}\right\}+\text { Const. }
\end{aligned}
$$

If $\Delta F_{0}$ at time $t, O_{2}(t)$, is represented by

$$
O_{2}(t)=\sum_{k=-M_{1}}^{M_{9}} w(k) O_{1}(t+k)
$$

where

$$
O_{1}(t)=0, \quad i<1 \text { or } i>l,
$$

and $w(k)$ is a weighted coefficient from $-M_{1}$ to $M_{2}$. Using Eq. (9) to Eq. (11), the partial derivatives with respect to $O_{1}(t)$ of the $\log$-likelihood $L$ can be computed. Finally, by equating the derivatives to zero, the following linear simultaneous equations are obtained:

$$
\left[\begin{array}{c}
\frac{\partial L}{\partial O_{1}(1)} \\
\frac{\partial L}{\partial O_{1}(2)} \\
\vdots \\
\frac{\partial L}{\partial O_{1}(l)}
\end{array}\right]=\boldsymbol{A}\left[\begin{array}{c}
O_{1}(1) \\
O_{1}(2) \\
\vdots \\
O_{1}(l)
\end{array}\right]=\boldsymbol{b},
$$

where $\boldsymbol{A}$ and $\boldsymbol{b}$ are an $l$-by- $l$ coefficient matrix and an $l$-dimensional column vector. A time series of $F_{0}, \boldsymbol{O}_{1}=\left[O_{1}(1), O_{1}(2), \ldots, O_{1}(l)\right]$, can be obtained by solving these equations. It can be also generated from multiple states in the same way.

\subsection{Other Remarks}

Further studies might investigate the following topics:

- The pitch-HMMs can also be utilized to select the best $F_{0}$ contour from several candidates in a database according to a maximum likelihood criterion.

- An automatic phonetic environment clustering can be performed by introducing the basic idea of the SSS algorithm. ${ }^{16)}$

\section{CONCLUSIONS}

In this paper, we have presented a new $F_{0}$ contour modeling using HMM and CMRT. In the proposed modeling, the $F_{0}$ contour of a sentence can be represented by normalized pitch patterns and maxima and dynamic ranges of minor phrases. This modeling is a sophisticated integration of the local statistical model (pitch-HMMs) represented by an HMM and the global statistical model (maximum and dynamic range models) represented by CMRT.

The experimental results for accent identification showed that the pitch-HMMs can express accent patterns appropriately and that $\Delta F_{0}$ was a useful parameter to generate reliable models. In the $F_{0}$ contour generation experiments for isolated words, the detailed models (15 phonemic categories) achieved an improvement of $10 \%$ for RMS error as compared with the rough models ( 2 categories). Even for the rough models, however, it was shown that sufficiently precise $F_{0}$ contours could be generated by the pitch-HMMs.

To generate the maximum and the dynamic range models, we used 11 and 10 factors, respectively. Particularly, in order to smooth a potential gap between minor phrases, preceding maximum and minimum for the factor of the maximum model and current maximum value for the factor of the dynamic range model were considered. As a result, suitable models were obtained; the measured multiple correlation coefficients of both models were 0.901 and 0.835 , respectively.

From the result of the subjective evaluation, the synthesized speech of the proposed $F_{0}$ contours was perceptually superior to that of the conventional model.

Through these experimental results, we showed that the proposed modeling was powerful and useful for a text-to-speech system. In this paper, we have adopted the simplest way for $F_{0}$ contour generation: fixed position alignment and straight line interpolation of the $F_{0}$ mean values. However, more sophisticated $F_{0}$ contours can be generated from the pitch-HMMs as discussed in section 6. As for future work, we will implement the proposed models into our text-to-speech system and investigate the expansion of the $F_{0}$ contour generation. 


\section{ACKNOWLEDGMENTS}

Acknowledgment is made to the Director of Media Technology Laboratory of Canon Inc. for permission to publish this paper. The authors also acknowledge the other members in their laboratory for participation in the experiments.

\section{REFERENCES}

1) T. Sakayori, S. Sasabe, and H. Kitagawa, "Prosody control using categorical multiple regression for speech synthesis by rule," Proc. Autumn Meet. Acoust. Soc. Jpn., 245-246 (1986) (in Japanese).

2) M. Abe and H. Sato, "Two-stage $F_{0}$ control model using syllable based $F_{0}$ units," Proc. ICASSP92, II.53-II.56 (1992).

3) K. Iwata and Y. Mitome, "Prosody generation models constructed by considering speech tempo influence on prosody," Proc. ICSLP92, 1155-1158 (1992).

4) T. Hirai, N. Iwahashi, N. Higuchi, and Y. Sagisaka, "Auto classification of $F_{0}$ control commands using statistical analysis," Tech. Rep. SP 94-12, IEICE (1994) (in Japanese).

5) K. Iwata, Y. Mitome, T. Seimitsu, M. Akamatsu, J. Kametani, K. Ozawa, and T. Watanabe, "Japanese text-to-speech conversion system using pitch controlled residual wave excitation method," Tech. Rep. SP 90-56, IEICE (1990) (in Japanese).

6) T. Yoshimura, S. Hayamizu, and K. Tanaka, "Word accent patterns modelling by concatenation of mora hidden Markov models," Proc. ICASSP 94, I.69-I.72 (1994).

7) S. Takahashi and S. Matsunaga, "Stochastic prosody modeling for accent phrase boundary detection in continuous speech," Tech. Rep. SP 90-71, IEICE (1990) (in Japanese).

8) A. Ljolje and F. Fallside, "Synthesis of natural sounding pitch contours in isolated utterances using hidden Markov models," IEEE Trans. Acoust. Speech Signal Process. 34, 1074-1080 (1986).

9) H. Sato, "Aanalysis of fundamental frequency characteristics related to phonemes," Proc. Autumn Meet. Acoust. Soc. Jpn., 259-260 (1989) (in Japanese).

10) S. Takeda, "A model for generating fundamental frequency contours considering phonemic fluctuation and rules for speech synthesis," Trans. IEICE, J73-A, 379-386 (1990) (in Japanese).

11) C. Hayashi, "On the quantification of qualitative data from the mathematicostatistical point of view," Ann. Inst. Math. 2 (1950).

12) Y. Sagisaka, K. Takeda, M. Abe, S. Katagiri, T. Umeda, and H. Kuwabara, "A large-scale Japanese speech database," Proc. ICSLP 90, 1089-1092 (1990).

13) X. D. Huang, Y. Ariki, and M. A. Jack, Hidden
Markov Models for Speech Recognition (University Press, Edinburgh, 1990).

14) S. Imai, "Log magnitude approximation (LMA) filter", Trans. IEICE, J63-A, 886-893 (1980) (in Japanese).

15) K. Hakoda and H. Sato, "Prosodic rules in connected speech synthesis," Trans. IEICE, J63-D, 715-722 (1980) (in Japanese).

16) J. Takami and S. Sagayama, "A successive state splitting algorithm for efficient allophone modeling," Proc. ICASSP 92, I.573-I.576 (1992).

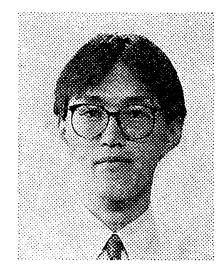

Toshiaki Fukada was born in Kyoto, Japan, in 1964 . He received B.E. and M.E. degrees from Tokyo Institute of Technology, Tokyo, Japan, in 1988 and 1990, respectively. In 1990, he joined Canon Media Technology Laboratories, Kawasaki Japan. Since 1995, he has been a researcher at ATR Interpreting Telecommunications Research Laboratories, Kyoto Japan. His research interests include spontaneous speech recognition, speech synthesis, and signal processing. He is a member of the Acoustical Society of Japan and the Institute of Electronics, Information and Communication Engineers.

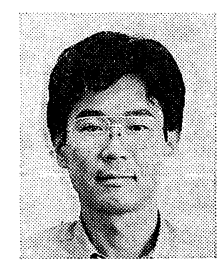

Yasuhiro Komori was born in Tokyo, Japan, in 1961. He received B.E. and M.E. degrees from Waseda University, Tokyo, Japan, in 1983 and 1985, respectively. In 1985, he joined Canon Inc. From 1988 to 1992, he was at ATR Interpreting Telephony Research Laboratories in Kyoto, Japan. In 1991, he was a visiting researcher at Carnegie Mellon University, U.S.A. From 1992, he is back to Canon Media Technology Laboratories, Kawasaki, Japan. He is an Associated Researcher at Canon. He received Kiyoshi Awaya Award from the Acoustical Society of Japan in 1991 fall-meeting. He received Dr. of Engineering degree from Waseda University in 1993. His research interests speech recognition and speech dialogue. He is a member of the Acoustical Society of Japan and the Institute of Electronics, Information and Communication Engineers.

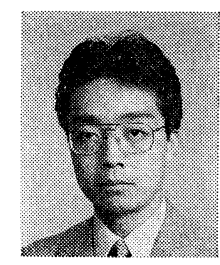

Takashi Aso was born in Tokyo, Japan, in 1960. He received B.E. and M.E. degrees from Nagoya Institute of Technology, Nagoya, Japan, in 1983 and 1986, respectively. From 1984 to 1985, he was a visiting researcher at Indiana University, U.S.A. In 1986, he joined Canon Inc. and is currently a researcher in Media Technology Laboratories, Kawasaki Japan. His research interests include speech synthesis, and signal processing. He is a member of the Acoustical 


\section{T. FUKADA et al: : STATISTICAL $F_{0}$ CONTOUR MODELING}

Society of Japan and the Institute of Electronics, Information and Communication Engineers.

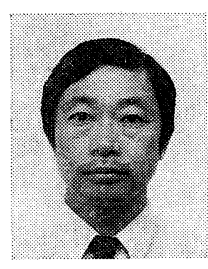

Yasunori Ohora was born in Toyohashi, Japan, in 1955. He received B.E. degree from Aoyama Gakuin University, Tokyo, Japan in 1976. In 1979, he joined Canon Inc. and is presently a group leader of speech processing group in Canon Media Technology Laboratories, Kawasaki Japan. His research interests include speech synthesis, and speech recognition. He is a member of the Acoustical Society of Japan. 